\title{
Design, development and applications of etched multilayers for soft X-ray spectroscopy
}

\author{
Karine Le Guen ${ }^{1,2, a}$, Rabah Benbalagh ${ }^{1,2}$, Jean-Michel André ${ }^{1,2}$, Jean-René Coudevylle ${ }^{3}$, and Philippe Jonnard ${ }^{1,2}$ \\ ${ }^{1}$ Sorbonne Universités, UPMC Université Paris 06, Laboratoire de Chimie Physique - Matière et Rayonnement, \\ 11 rue Pierre et Marie Curie, 75231 Paris Cedex 05, France \\ 2 CNRS UMR 7614, Laboratoire de Chimie Physique - Matière et Rayonnement, 11 rue Pierre et Marie Curie, \\ 75231 Paris Cedex 05, France \\ ${ }^{3}$ Institut d'Électronique Fondamentale, Université Paris-Sud, CNRS, 91405 Orsay Cedex, France
}

Received: 22 July 2016 / Received in final form: 22 March 2017 / Accepted: 20 April 2017

(c) EDP Sciences 2017

\begin{abstract}
An etched multilayer, a 2D structure fabricated by etching a periodic multilayer according to the pattern of a laminar grating, is applied in the soft X-ray range to improve the spectral resolution of wavelength dispersive spectrometers. The present article gathers all the successive stages of the development of such a device optimized to analyze the characteristic emission of light elements: design, structural and optical characterization and applications to X-ray spectroscopy. The evolution of the shape of the $\mathrm{C}$ K $\alpha$ emission band of highly oriented pyrolytic graphite (HOPG), as a function of the angle between the emission direction and the $(0001)$ planes, is measured. These results, compared to those with a grating, demonstrate that the achieved spectral resolution enables disentangling $\sigma \rightarrow 1 s$ and $\pi \rightarrow 1 s$ transitions within the $\mathrm{C} \mathrm{K}$ emission band.
\end{abstract}

\section{Introduction}

Although high spectral resolution is currently achieved on grating X-ray spectrometers installed at the end-station of synchrotron beamlines, it remains a topic of interest for laboratory apparatus like spectrometers, electron microprobes or in wavelength-dispersive spectrometer (WDS) on scanning electron microscope (SEM), which mainly rely on crystal spectrometers. The high resolution enables being sensitive to the chemical environment of the emitting atoms (pure element vs. oxide, pure layers vs. diffusion interlayers in stratified samples, etc.) and improve the quantitative analysis and limit of detection (less overlapping of close emissions and decrease of the continuous background).

Dispersing X-ray radiation requires a structure presenting a period of the order of the radiation wavelength. Thus since the beginning of the 20th century crystals are used for that purpose $[1,2]$. However, if the wavelength is too large, in the extreme UV (EUV) or ultra-soft X-ray ranges $(30-500 \mathrm{eV})$, it is not possible to find crystals having sufficiently large plane spacing to fulfill the Bragg law. Thus, pseudo-crystal presenting a large period have been developed then followed by periodic multilayers [3].

\footnotetext{
a e-mail: karine.le_guen@upmc.fr
}

Periodic multilayers play a crucial role for optical applications (X-ray microscopy, EUV lithography, space telescopes, synchrotron beamlines) from the EUV to the hard $\mathrm{X}$-ray ranges. The width of the diffraction pattern of the dispersive element is generally the factor limiting the spectral resolution. To give an order of magnitude, in the C K emission photon energy range $(280 \mathrm{eV})$, since the diffraction pattern can be as large as $25-30 \mathrm{eV}$, the spectral resolution is poor.

In order to enhance the spectral resolution, the pioneering idea of André [4-7] was to lighten the multilayer structure by introducing some emptiness within the stack according to the profile of a lamellar grating. Since absorption by the multilayer is then reduced, the incoming radiation penetrates more deeply within the structure: consequently, a higher number of bilayers takes part to the Bragg diffraction leading to narrower Bragg peaks. Highresolution X-ray spectrometers are required to disentangle close emission lines, improve the detection limit and study the shape of emission bands related to the chemical state of the emitting atoms [8-11]. Let us note that another possibility to obtain such structures is to deposit a multilayer onto a grating substrate $[12,13]$. In X-ray spectroscopy a compromise between intensity and resolution has to be made. Commercial apparatus, most of the time designed for quantitative analysis, put emphasis on the intensity 
at the detriment of the spectral resolution. Laboratory and synchrotron apparatus, designed for applied or fundamental physics, give preference to the resolution. The etched multilayer can fill the gap between commercial and laboratory apparatus, owing to the optimized reflectance coming from the multilayer structure and the optimized resolution coming from the grating structure.

The present article gathers the successive stages of the development of an etched multilayer optimized for the spectroscopy of light elements. The design of the etched mirror is optimized as a compromise between calculated reflectance and bandwidth. Starting from a multilayer, the optimal etched structure is fabricated with the microelectronics tools and recipes. Then, SEM and soft X-ray reflectivity measurements are performed to characterize the dimensions and the optical properties of the etched multilayer respectively. In order to estimate the gain of resolution provided by the etching, the multilayer mirror and etched multilayer are successively inserted in two types of spectrometers (plane- and curved-crystal). Finally, to test the spectral resolution of the etched multilayer in the case of slight changes of the shape of an emission band, compared to that of a grating, the anisotropic $\mathrm{C} \mathrm{K} \alpha$ emission originating from highly oriented pyrolytic graphite (HOPG) is chosen as a suitable candidate.

The multilayer, made of the periodic alternation of thin layers of two- or more- materials deposited onto a substrate, acts as a mirror according to the Bragg diffraction law. When dedicated to the X-EUV spectral domain, layers are of nanometric thickness. The period of the stack is labeled $d$.

As illustrated on Figure 1, the etched multilayer exhibits a double periodicity: in addition to the period $d$ of the multilayer, the period $D$ of the grating (alternation of bars of multilayer and groove of given thickness) has to be considered. The etched mirror is used at the 0 th order of diffraction by the grating and at the 1st (or the 2nd) order of diffraction by the multilayer. In the following, MM will stand for "multilayer mirror" while EM will correspond to "etched multilayer". The spectral resolution gain is close to the $\Gamma$ factor of the EM, $\Gamma$ being the ratio of the multilayer bar thickness to the grating period $D$. Another important advantage of the EM is the reduction of the total specular reflection. With respect to the corresponding MM, the total reflection domain of the EM is reduced since the critical angle is diminished by a factor $\sqrt{\Gamma}$, resulting in an improvement of the detection limit [14].

\section{Design and fabrication}

\subsection{Design}

Given a Mo/B ${ }_{4} \mathrm{C}$ stack made of 300 bilayers (period $d=$ $6 \mathrm{~nm}$, total height $\sim 1.8 \mu \mathrm{m}$ ), the optimization of the structural parameters of the etched multilayer is achieved through the computation of the diffraction pattern as a function of the grazing angle in the boron $\mathrm{K}$ emission region $(\sim 180 \mathrm{eV})$. The calculation is carried out by means

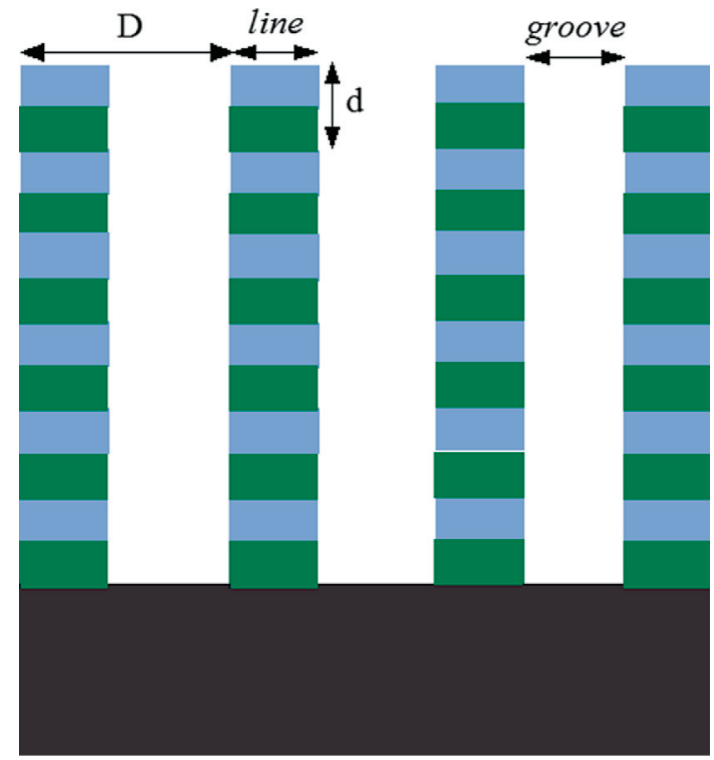

Fig. 1. Scheme of a multilayer etched according to a rectangular profile. The multilayer and grating periods are labeled $d$ and $D$ respectively. The meaning of "line" and "groove" is also depicted.

of the modal method $[5,6,15,16]$ in which, for a given value of $\Gamma$ a compromise between reflectivity and bandwidth has to be found. On one hand, with respect to the non-etched multilayer MM, introducing in a periodic manner columns of void within the stack leads to a significant reduction of the width of the Bragg peaks. On the other hand, owing to the lower area of the dispersive element, the reflectance of the mirror decreases. To enhance the spectral resolution without a large loss of peak reflectivity, the width of the grating lines is set to $350 \mathrm{~nm}$, these lines being separated by $650 \mathrm{~nm}$. From the calculations, compared to the non-etched structure, the bandwidth is expected to be reduced by a factor close to 2.5 while the reflectance loss is about $33 \%$. Other calculation methods are possible: coupled wave analysis [17] or unified analytical theory [18].

\subsection{Fabrication}

The fabrication of the etched multilayer relies, in terms of techniques and recipes, on microelectronic processes. A schematic view of the different fabrication steps is depicted in Figure 2. Despite the calculations were performed for 300 bilayers, during fabrication the considered MM was made of only 200 bilayers in order to limit the overall aspect ratio of the structure to prevent possible delamination. The positive photoresist (UV III), deposited on the $\mathrm{SiO}_{2}$ layer on the top of the $\mathrm{Mo} / \mathrm{B}_{4} \mathrm{C}$ stack by spin coating, is exposed to a deep-UV radiation $(248 \mathrm{~nm})$ through a mask on which the desired pattern (in our case, lines of given width and spacing) has been previously printed. During the development step, parts of resist that have been insolated are dissolved: lines on non-insolated 


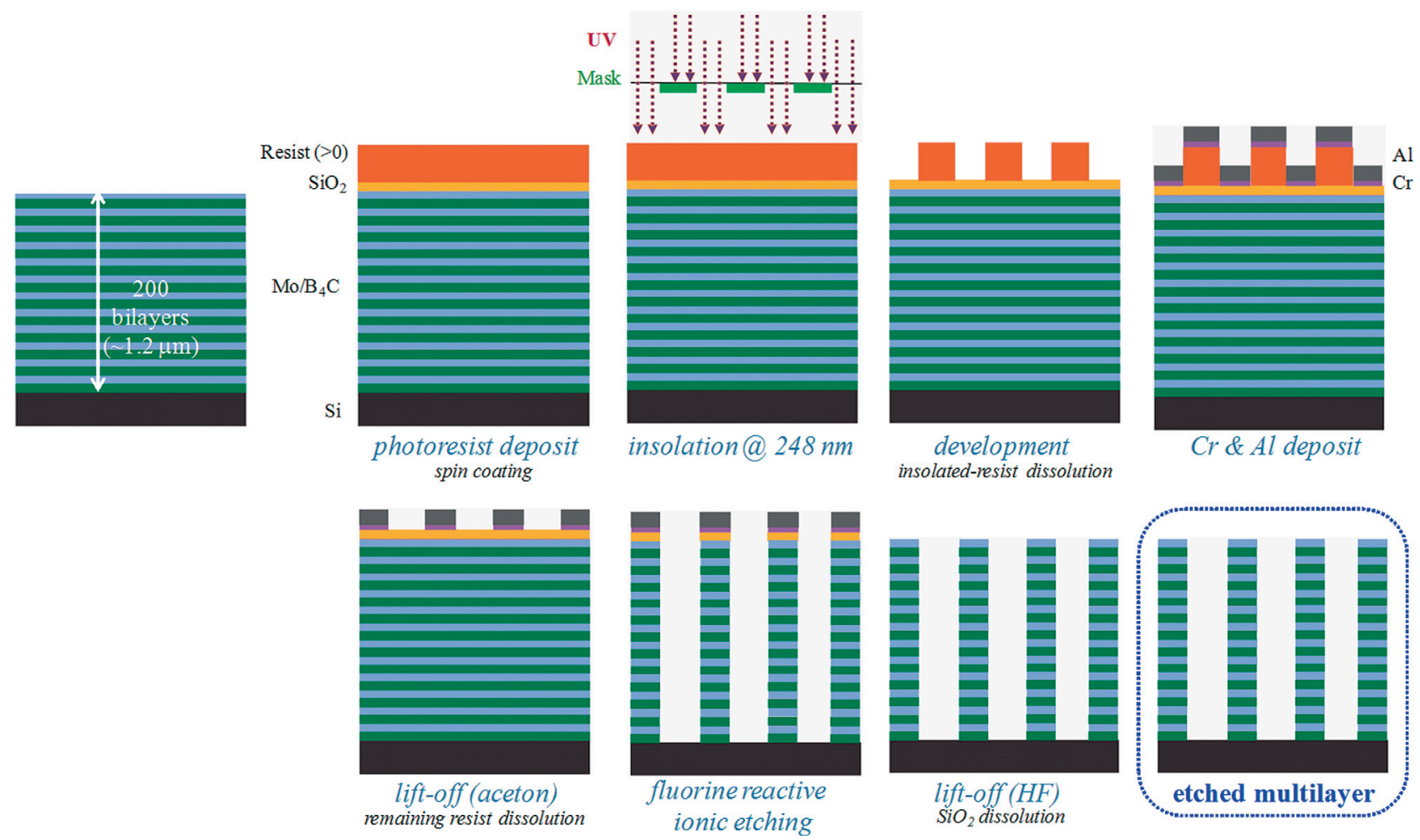

Fig. 2. Schematic view of the fabrication process of an etched multilayer.

resist remain on the top of the multilayer. Following a deposit of $\mathrm{Cr}(\sim 5 \mathrm{~nm})$ and $\mathrm{Al}(\sim 60 \mathrm{~nm})$ and a lift-off bath to remove the remaining resist, the mask pattern is now transferred onto the top of the multilayer. These series of $\mathrm{Al}$ lines acts as a mask during the following step, namely the highly-directional reactive ion etching (RIE) involving fluorinated species. Finally, the remaining $\mathrm{Al}$ lines are removed by dissolution of $\mathrm{SiO}_{2}$ in a $\mathrm{HF}$ bath.

\section{Characterization}

Prior application to WDS as the dispersive element, characterization of the EM is mandatory. First, the effective dimensions of the grating have to be estimated and compared to their nominal values: this constitutes the final validation of the implemented recipe. Then, the optical performances of the etched multilayer (reflectance and bandwidth) in the spectral region of interest have to be measured and compared to (i) those measured for the nonetched stack and (ii) those calculated during the design step.

\subsection{SEM images}

Owing to the sub-micrometric dimensions of the grating patterned into the multilayer, electron microscopy is required to check the values of the structural parameters (width and spacing of the grating lines) as well as estimate the homogeneity of the etching process on the overall sample. SEM images are presented on Figure 3. On an extended area, very regular "bars of multilayers", separated by a rather rough bottom of the grating, are "drawn". Owing to the tilt angle of the etched multilayer sample, the estimation of the dimensions has to be considered as rather approximate: measured values are close to those fixed at the end of the structure optimization step.

\subsection{Reflectance at the application wavelength}

Once the grating structure of aimed dimensions is validated, the optical properties of the etched multilayer mirror have to be tested. Figure 4 presents the comparison, in the boron and carbon $\mathrm{K}$ spectral regions (180 and $277 \mathrm{eV}$ respectively [19]), of the reflectivity curves of the MM and the EM structures measured at the 1st order of diffraction on the BEAR beamline at the Elettra synchrotron facility. For the EM, an intensity scaling factor is applied in both ranges. For sake of comparison, all these measured values are listed in Table 1, in addition with those calculated during the design step in the B $\mathrm{K}$ emission range [16].

For both energies, the profile of the MM curve is made of an unique Bragg peak while that of the EM exhibits a main peak (1st order of diffraction by the multilayer) surrounded by secondary peaks of variable intensities coupling with the grating diffraction orders). The reflectance of the EM is close to $8 \%$ at $180 \mathrm{eV}$ and 
The European Physical Journal Applied Physics

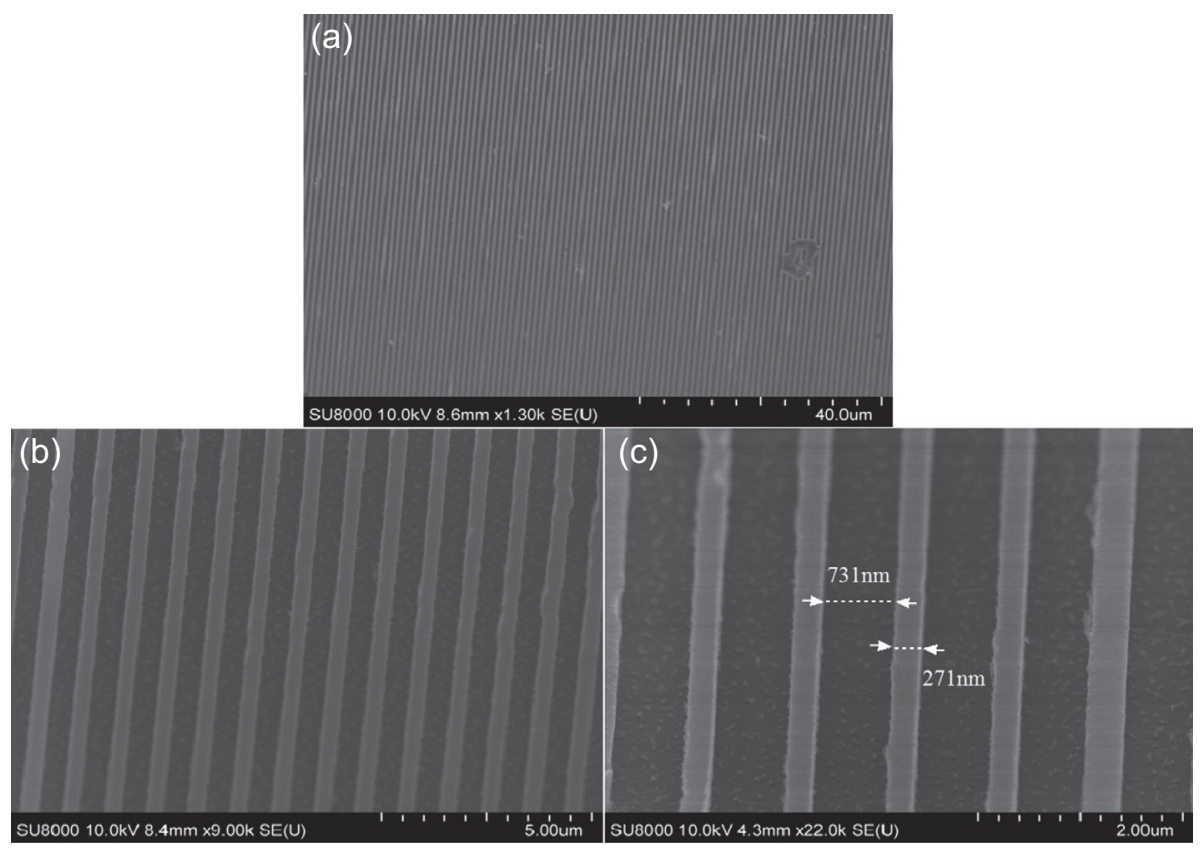

Fig. 3. SEM images of an etched multilayer: overall view (a) and (b) and estimated dimensions (c).

(a)

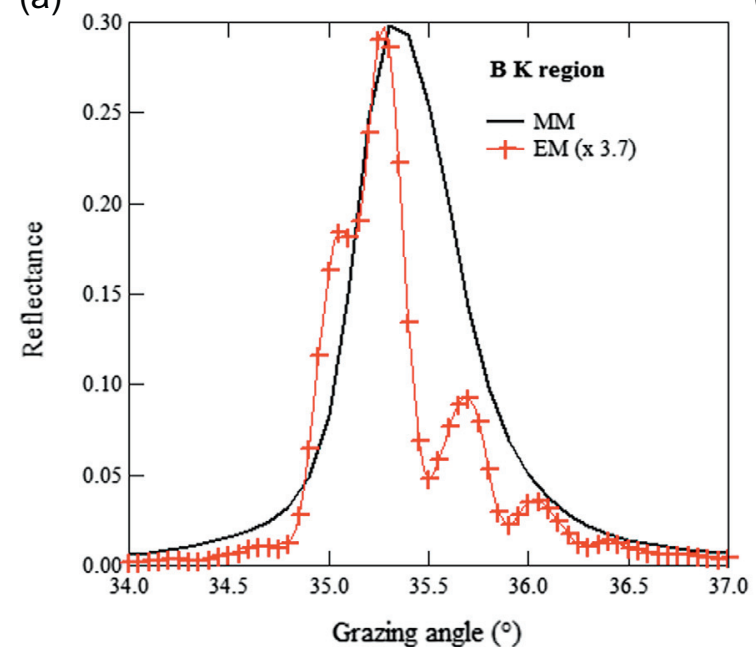

(b)

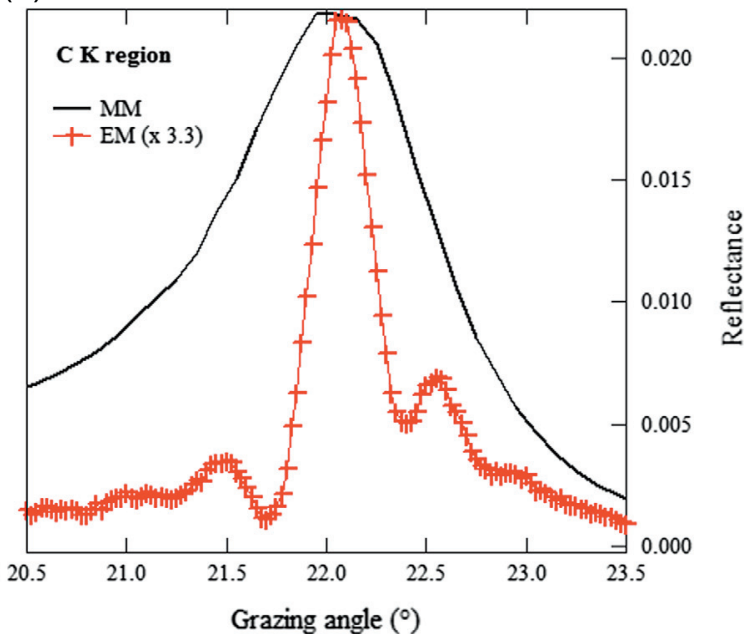

Fig. 4. Comparison, in the B K $(180 \mathrm{eV}$, a) and $\mathrm{C} \mathrm{K}(277 \mathrm{eV}, \mathrm{b})$ regions, of the reflectivity curves of the MM and EM structures. For the EM, an intensity scaling factor equal to 3.7 and 3.3 is applied in the boron and carbon ranges respectively.

Table 1. Comparison of the calculated and measured values of the reflectance $R(\%)$ and full width at half maximum (FWHM, ${ }^{\circ}$ and $\mathrm{eV}$ ) of the first Bragg peak of the MM and EM in the spectral ranges of interest. During the design step, calculations were performed in the B $\mathrm{K}$ emission range.

\begin{tabular}{|c|c|c|c|c|c|c|c|}
\hline \multirow[t]{2}{*}{ Spectral range } & \multirow[t]{2}{*}{ Physical value } & \multicolumn{3}{|c|}{$\begin{array}{l}\text { Caculations during design } \\
\text { (from Ref. [16]) }\end{array}$} & \multicolumn{3}{|c|}{ Experimental } \\
\hline & & MM & EM & Ratio MM/EM & MM & EM & Ratio MM/EM \\
\hline \multirow[t]{3}{*}{ B K emission $\sim 180 \mathrm{eV}$} & Reflectance $R(\%)$ & 46.7 & 31.3 & 1.5 & 29.9 & 8.0 & 3.7 \\
\hline & FWHM $\left(^{\circ}\right)$ & 0.77 & 0.31 & 2.5 & 0.59 & 0.41 & 1.4 \\
\hline & FWHM (eV) & 3.45 & 1.4 & 2.4 & 2.3 & 1.8 & 1.3 \\
\hline \multirow[t]{3}{*}{ C K emission $\sim 277 \mathrm{eV}$} & Reflectance $R(\%)$ & - & - & - & 2.2 & 0.65 & 3.4 \\
\hline & FWHM $\left(^{\circ}\right)$ & - & - & - & 1.4 & 0.34 & 4.1 \\
\hline & FWHM (eV) & - & - & - & 14.1 & 4.2 & 3.4 \\
\hline
\end{tabular}


$0.7 \%$ at $277 \mathrm{eV}$. Keeping in mind that the EM structure has been optimized for the B K domain, as expected the reflectance values are higher at $180 \mathrm{eV}$ than at $277 \mathrm{eV}$ for both types of multilayers. The peak reflectivity loss due to etching can be estimated to be around $70 \%$ in both spectral regions. This value is twice higher than that predicted by the calculations.

The comparison between calculated and measured values of the peak reflectivity is not obvious since defects as interfacial roughness degrade the nominal value. This explains the discrepancy between calculated and measured reflectance values even in the case of the MM (loss of $33 \%$ ). The etching process introduces other types of imperfection: dimension loss, interdiffusion following annealing during silica deposition, roughness of the bar sides, etc. The narrowing of the bandwidth upon etching in the $\mathrm{B} \mathrm{K}$ region is evaluated to be only about $1.3-1.4$, which is about half the value predicted by the calculations at $\sim 180 \mathrm{eV}$. Indeed, in the case of the EM, the secondary peak located at $\sim 35^{\circ}$ exhibiting an intensity higher than the half maximum of the main peak, the full width at half maximum (FWHM) of the first Bragg peak is broadened. In the $\mathrm{C} \mathrm{K}$ region, upon etching, the width of the first Bragg peak is reduced by a factor 3.4-4.1. This order of magnitude can be related to the reverse of the $\Gamma$ value. Finally we have checked that, for a given EM sample, the reflectivity curve of the EM is reproducible when the incident radiation spot is shifted on different locations of the EM surface.

Recently, the coupled-mode theory was implemented to calculate the diffraction pattern of the EM as a function of the shape (rectangular or trapezoidal) of the etching profile for different values of $\Gamma$ [20]: these calculations show that (i) the secondary peaks observed in Figure 4 originate from the coupling of the 1st order of diffraction by the multilayer with the orders of diffraction by the grating (ii) the relative intensity of the secondary peaks depends on the shape of the lines of the grating. The limited gain in bandwidth $(\sim 1.5)$ in the B K region can be improved by diminishing the period $D$ of the grating (the grating diffraction orders would then be better separated from the 1st multilayer diffraction order [21]) and by a better control of the rectangular patterning of the grating lines. Reducing the value of $D$ (for example from $1 \mu \mathrm{m}$ to $300 \mathrm{~nm}$ with a $90 \mathrm{~nm}$-wide lines) leads to the complete change of the fabrication process (for example nano-imprint or electron lithography instead of deep UV lithography), keeping in mind that the lower the grating dimensions are, the more difficult the control of the patterning is.

\section{Applications in X-ray spectroscopy}

The EM will now be implemented in a WDS to act as the dispersive element monochromatizing the polychromatic $\mathrm{X}$-ray radiation (produced by an X-ray tube), according to the Bragg diffraction law.

\subsection{Insertion in a plane spectro-goniometer}

The EM is first inserted in the MONOX goniometer [22] used as a plane spectrometer operating in the WDS mode. A boron carbide $\left(\mathrm{B}_{4} \mathrm{C}\right)$ thin film deposited onto a silicon substrate is used as the target in the X-ray tube. The polychromatic X-ray radiation emitted by the sample enters the goniometer chamber where the multilayer (MM or EM) on the sample holder disperses the radiation in the $\theta-2 \theta$ mode towards the Geiger-Muller (GM) counter. The comparison of the X-ray spectrum recorded with the MM or the EM is depicted in Figure 5.

Both spectra exhibit the $\mathrm{B}$ and $\mathrm{C} \mathrm{K} \alpha$ emissions at 183 and $280 \mathrm{eV}$ respectively as well as the $\mathrm{O} \mathrm{K} \alpha$ emission around $525 \mathrm{eV}$ indicating the oxidation of the $\mathrm{B}_{4} \mathrm{C}$ film. This last emission gives rise to a large asymmetrical peak using the MM while, when applying the EM, results in the improvement of both resolution and detection limit, the structure now reveals the $\mathrm{Si} \mathrm{K} \alpha$ emission at the $3 \mathrm{rd}$ and 4th diffraction orders. As observed here, the detection limit is lowered by at least a factor 2 since it varies as the square root of the background intensity [23]. The amelioration of the spectral resolution is clearly seen by the disentanglement of the $\mathrm{C} \mathrm{K} \alpha$ (1st order of diffraction) and $\mathrm{O} \mathrm{K} \alpha$ (2nd order) emissions.

\subsection{Insertion in a high-resolution curved-crystal Johann-type spectrometer}

The EM is now inserted in the IRIS (instrument for research on interfaces and surfaces) concave bent-crystal Johann-type spectrometer [24]. In this apparatus, to ensure the high spectral resolution, the (virtual) source, the crystal and the detector stay on the focusing Rowland circle which radius is equal to $250 \mathrm{~mm}$. The first step consists in the curvature of the multilayer (MM and EM) according to a radius $500 \mathrm{~mm}$ by inserting the device (MM and EM) in the curved crystal-holder of the spectrometer. Owing to the large curvature radius $(500 \mathrm{~mm})$ with respect to the total thickness of the sample $(400 \mu \mathrm{m}$-thick Si substrate and $1.2 \mu \mathrm{m}$-thick etched mirror), the mechanical constraint on the substrate is small and no fracture of the substrate or delamination of the stack occurred. Furthermore, we assume that the curvature does not affect the line-to-space ratio.

The characterization of the EM (Sect. 3.2) has shown that unfortunately, although optimized for the B K domain, the structure does not allow a significant reduction of the width of the Bragg peak in that region due to the coupling of the 1st order of diffraction by the multilayer with the orders of diffraction by the grating [20]. On the other hand, in the $\mathrm{C} \mathrm{K}$ range, despite a low reflectance $(0.65 \%)$, the gain in spectral resolution is valuable. Consequently, in the following, only $\mathrm{C} \mathrm{K} \alpha$ emissions originating from a $\mathrm{SiC}$ thin film and graphite bulk sample will be considered. Each of these samples is used as the target bombarded by energetic electrons $(8 \mathrm{keV})$. The emitted X-rays enter the spectrometer chamber through a slit (virtual source), are diffracted by the curved-multilayer 
The European Physical Journal Applied Physics

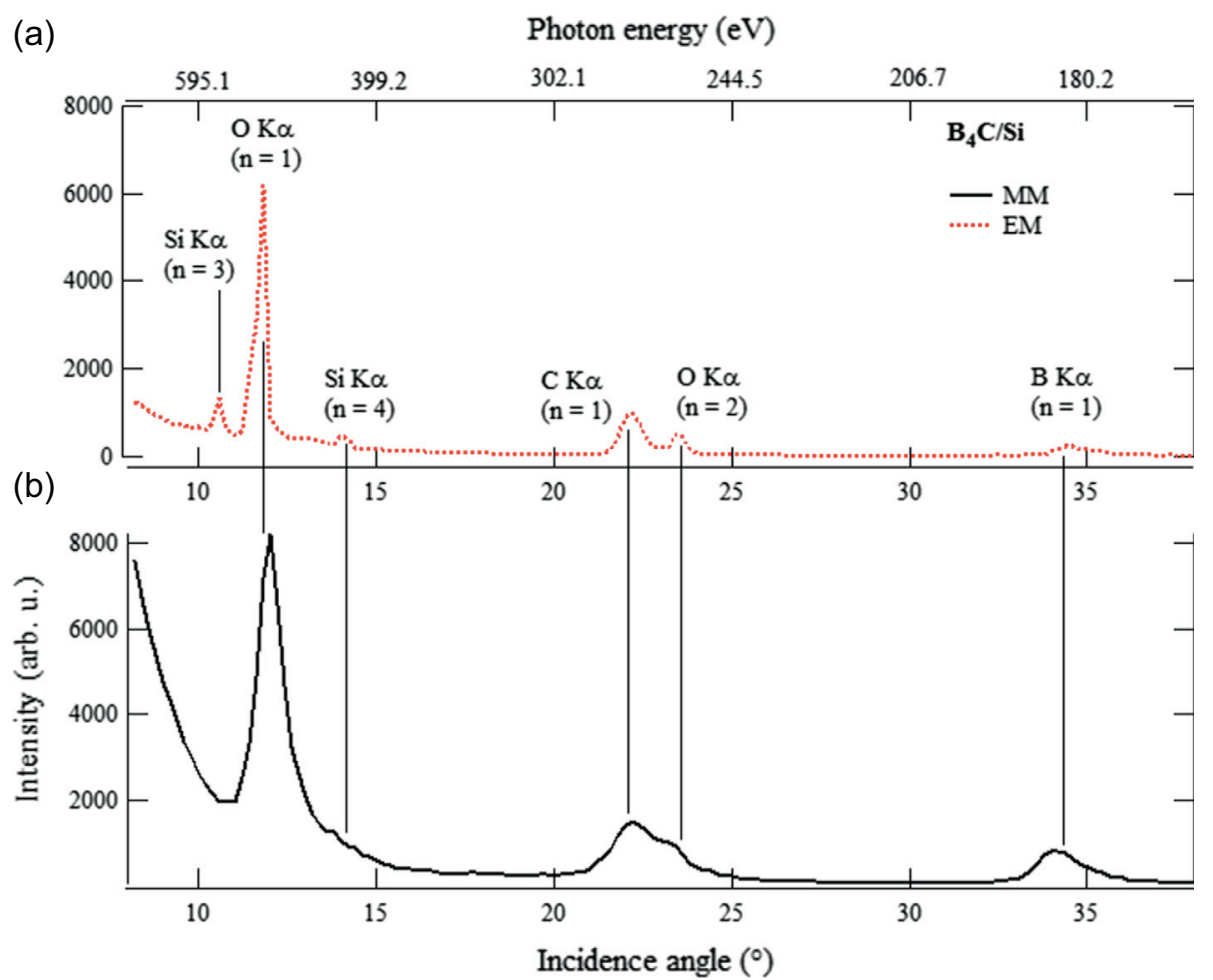

Fig. 5. Comparison of the X-ray spectrum of a $\mathrm{B}_{4} \mathrm{C}$ thin film deposited onto a silicon substrate obtained using a EM (a) or a MM (b) acting as the dispersive element of a plane spectrometer.

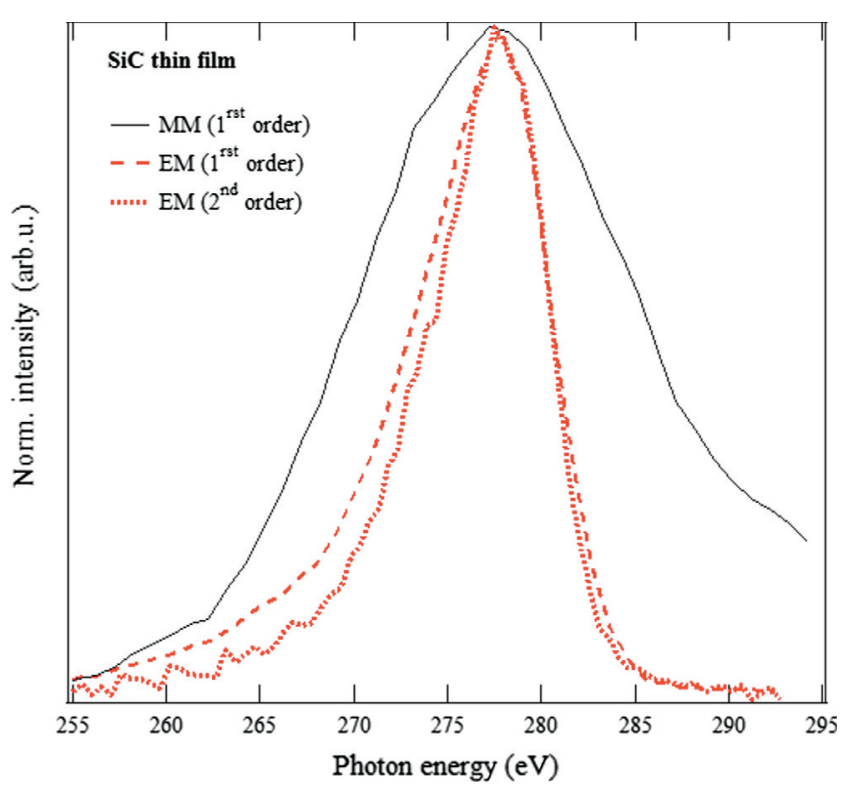

Fig. 6. Comparison of the $\mathrm{C} \mathrm{K} \alpha$ emission band of a $\mathrm{SiC}$ thin film recorded using a curved MM or EM acting as the dispersive element in a bent-crystal spectrometer.

(MM or EM) in the $\theta-2 \theta$ mode towards a GM counter. The low peak reflectivity of the multilayer in this region is counterbalanced by a high number of scans leading to reliable spectra.

The $\mathrm{C} \mathrm{K} \alpha$ emission band of the SiC thin film deposited onto $\mathrm{Si}$ and recorded with the $\mathrm{MM}$ or the EM is presented in Figure 6. The spectra are normalized to their maximum. With respect to the MM, operating the EM at the 1st diffraction order leads to a bandwidth divided by a factor 1.9. This value is lower than the experimental MM/EM width ratio (equal to 3.4 ) presented in Table 1. This discrepancy comes from the natural bandwidth (about $10 \mathrm{eV}$ for the emission bands of light elements such as carbon) which has also to be taken into account to explain the value of the observed width. Operating the EM at the 2nd diffraction order reinforces the width reduction which is then close to a factor 2.4 compared to the use of the MM.

The anisotropic $\mathrm{C} \mathrm{K} \alpha$ emission band originating from HOPG constitutes an efficient test to probe the spectral resolution of a specific dispersive device or an entire spectrometer $[25,26]$. We take advantage of the improved resolution when operating the EM at the 2nd diffraction order to evidence the slight modifications of the shape of the $\mathrm{C} \mathrm{K} \alpha$ band as a function of the angle $\delta$ between the emission direction and the orientation of the (0001) planes (or take-off angle). The angular dependence of the intensity of the $\mathrm{C} \mathrm{K} \alpha$ emission band is caused by the different symmetries of the initial orbital involved in the electron transition. The total emission intensity $I(\delta, E)$ is written as [27]:

$$
I(\delta, E)=\cos ^{2} \delta \times I_{\pi}^{0}(E)+\frac{1}{2}\left(1+\sin ^{2} \delta\right) \times I_{\sigma}^{0}(E),
$$

where $I_{\pi}^{0}(E)$ and $I_{\sigma}^{0}(E)$ stand for the maximum intensities from the $\pi$ - and $\sigma$-bonding orbitals respectively. 
(a)

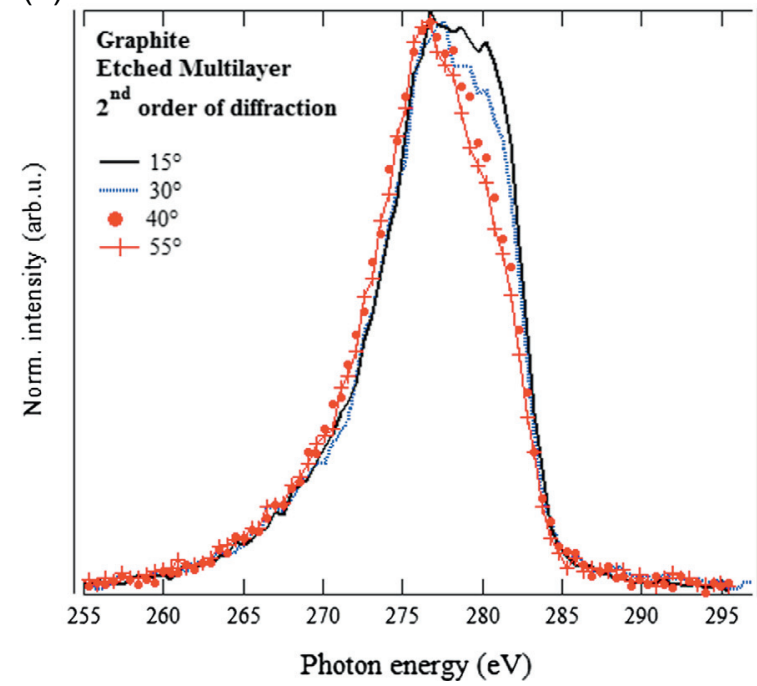

(b)

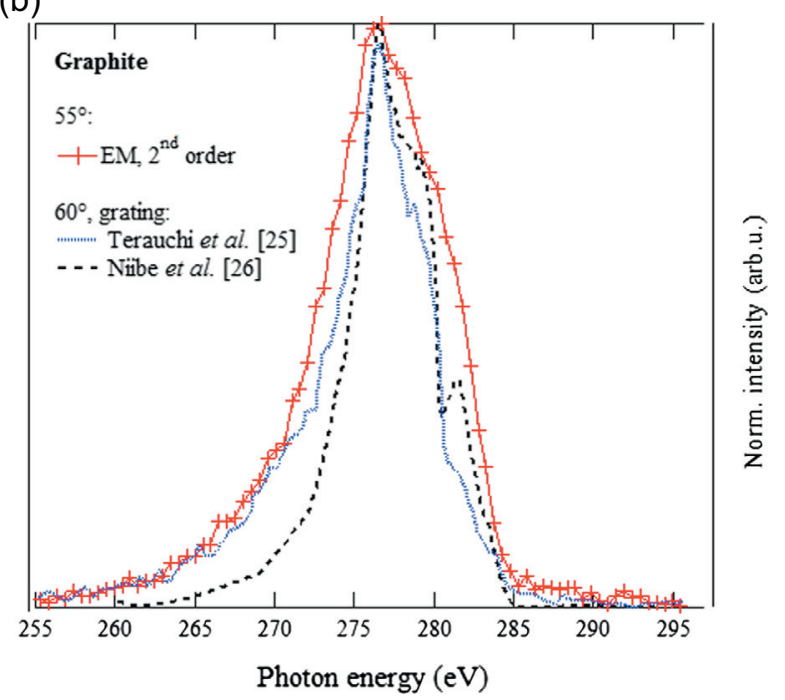

Fig. 7. Evolution of the shape of the $\mathrm{C} \mathrm{K} \alpha$ emission band of HOPG as a function of the angle between the emission direction and the orientation of the $(0001)$ planes (a) and comparison of the $\mathrm{C} \mathrm{K \alpha}$ emission band recorded with the EM operated at the second diffraction order and a grating spectrometer [25] and [26] (b).

The $\mathrm{C} \mathrm{K} \alpha$ emission band of graphite is recorded for different angle values between the emission direction and the orientation of $\left(\begin{array}{lll}0 & 0 & 0\end{array}\right)$ planes using the EM. The evolution of the shape of the $\mathrm{C} \mathrm{K} \alpha$ band is shown on the left part of Figure 7 . The spectra are normalized to their maximum. When these two directions are quite close $\left(15^{\circ}\right)$, the top of the band presents an intensity plateau about $4 \mathrm{eV}$ wide. Then, as this angle increases, the intensity of the high-energy side of the band is significantly decreased. The intensity of the $\pi \rightarrow 1 s$ transition $(\sim 282 \mathrm{eV})$ relative to the intensity of the $\sigma \rightarrow 1 s$ transition $(\sim 277 \mathrm{eV})$ is sensitive to the emission angle. The EM is here able to discriminate these two types of transitions.

To end, it is useful to compare the EM performances with those of the dispersive element insuring the best spectral resolution in this energy region, namely a grating. With this latter, since diffraction occurs at the surface of the grating, absorption is not limiting the spectral resolution which can reach a few thousands $(\mathrm{E} / \Delta \mathrm{E})$ in that photon energy range. On the right part of Figure 7, for the largest angle $\left(55^{\circ}\right)$, the $\mathrm{C} \mathrm{K} \alpha$ emission band recorded with the EM at the 2nd diffraction order is compared to that measured at $60^{\circ}$ with a grating spectrometer by Terauchi et al. [25] and very recently by Niibe et al. [26] The three spectra are aligned according to the position of their maximal intensity. In the case of the EM, the contribution of the $\pi \rightarrow 1 s$ transition is higher than that recorded with the grating since the emission angle is lower (the $60^{\circ}$ value was not possible due to mechanical constraints). The EM spectrum compares well with the two grating spectra but is broader. It is obvious that the EM cannot act better than a grating but brings the same chemical information and thus its application to X-ray spectroscopy is of valuable interest. In summary, EM appears as a good compromise between MM implemented in curved-crystal and grating spectrometers.

\section{Conclusions}

We have designed and fabricated $\mathrm{Mo} / \mathrm{B}_{4} \mathrm{C}$ multilayers etched upon a lamellar grating profile. At the $\mathrm{B} \mathrm{K}$ energy, the EM reflectance is measured to be $8.0 \%$ while the improvement of the spectral resolution upon etching is about half the value predicted by the calculations. At the C K energy, despite a poor reflectance of the EM (0.65\%), the reduction of the bandwidth upon etching by a factor higher than 3 is of valuable interest for spectroscopy since it allows probing the shape of characteristic C K $\alpha$ emission bands to discriminate different chemical states. The resolution achieved with the EM, with respect to that of a grating, has been shown in the case of the anisotropic $\mathrm{C} \mathrm{K} \alpha$ emission in HOPG where $\sigma \rightarrow 1 s$ and $\pi \rightarrow 1 s$ transitions can be distinguished.

To overcome the limitations in the $\mathrm{B} \mathrm{K}$ region, the design of the EM has to be modified. In order to properly separate the multilayer and grating diffraction orders, the coupling of these latter being critical in this domain, the value of the grating period $\mathrm{D}$ has to be reduced (for example from $1 \mu \mathrm{m}$ to $300 \mathrm{~nm}$ but keeping the same $\Gamma$ value). It is obvious that the subsequent reduction of the width of the grating lines renders more difficult the production process. To enhance the reflectance in the $\mathrm{C} \mathrm{K}$ region, a better control of the EM dimensions during the patterning process of the MM is required. Ideally, a new EM has to be designed (choice of the suitable combination of materials, reduction of the grating period, etc.).

The EM, which design is made as a compromise between resolution and reflectance, allows spectroscopic applications with performances intermediate between those of multilayer- and grating-based spectrometers.

N. Isac from IEF is acknowledged for help during EM fabrication as well as the team of the BEAR beamline from Elettra for 
support during reflectivity measurements. Incoatec is thanked for providing us with $\mathrm{Mo} / \mathrm{B}_{4} \mathrm{C}$ multilayers.

\section{References}

1. E.P. Bertin, Principles and Practice of X-Ray Spectrometric Analysis (Plenum Press, New York, 1970)

2. C. Bonnelle, C. Mandé, Advances in X-Ray Spectroscopy (Pergamon Press, Oxford, 1982)

3. E. Spiller, Appl. Phys. Lett. 20, 365 (1972)

4. H. Berrouane, J.-M. André, R. Barchewitz, C. Khan Malek, R. Rivoira, Opt. Comm. 76, 111 (1990)

5. B. Pardo, J.-M. André, A. Sammar, J. Opt. 22, 141 (1991)

6. A. Sammar, J.-M. André, B. Pardo, Opt. Commun. 86, $245(1991)$

7. A.-E. Sammar, J.-M. André, M. Ouahabi, B. Pardo, R. Barchewitz, C. R. Acad. Sci., Sér. 2: Sci. Terre Planètes 316, 1055 (1993)

8. P. Jonnard, C. Bonnelle, K. Danaie, A. Bosseboeuf, E. Beauprez, Surf. Interface Anal. 29, 255 (2000)

9. I. Jarrige, P. Holliger, P. Jonnard, Thin Solids Films 458, $314(2004)$

10. H. Maury, P. Jonnard, J.-M. André, J. Gautier, F. Bridou, F. Delmotte, M.-F. Ravet, Surf. Sci. 601, 2315 (2007)

11. P. Jonnard, K. Le Guen, R. Gauvin, J.-F. Le Berre, Microsc. Microanal. 15, 36 (2009)

12. D.L. Voronov, E. H. Anderson, R. Cambie, P. Gawlitza, L.I. Goray, E.M. Gullikson, F. Salmassi, T. Warwick, V. Yashchuk, H.A. Padmore, J. Phys.: Conf. Ser. 425, 152006 (2013)

13. F. Choueikani, F. Delmotte, F. Bridou, B. Lagarde, P. Mercere, E. Otero, P. Ohresser, F. Polack, J. Phys.: Conf. Ser. 425, 152007 (2013)
14. M. Fialin, H. Rémy, J.-M. André, J.-P. Chauvineau, F. Rousseaux, M.-F. Ravet, D. Decanini, E. Cambril, X-Ray Spectrom. 25, 60 (1996)

15. R. Benbalagh, J.-M. André, R. Barchewitz, P. Jonnard, G. Julié, L. Mollard, G. Rolland, C. Rémond, P. Troussel, R. Marmoret, E.O. Filatova, Nucl. Instrum. Methods Phys. Res. A 541, 590 (2005)

16. R. Benbalagh, Ph.D. thesis, Université Pierre et Marie Curie, Paris, 2003

17. I.V. Kozhevnikov, R. van der Meer, H.M.J. Bastiaens, K.-J. Boller, F. Bijkerk, Opt. Express 18, 16234 (2010)

18. X. Yang, I.V. Kozhevnikov, Q. Huang, Z.S. Wang, J. Opt. Soc. Am. B 32, 506 (2015)

19. P. Jonnard, C. Bonnelle, X-ray Spectrom. 40, 12 (2011)

20. J.-M. André, K. Le Guen, P. Jonnard, X-ray Spectrom. 43, $122(2013)$

21. R. van der Meer, I. Kozhevnikov, B. Krishnan, J. Huskens, P. Hegeman, C. Brons, B. Vratzov, B. Bastiaens, K. Boller, F. Bijkerk, AIP Adv. 3, 012103 (2013)

22. J.-M. André, A. Avila, R. Barchewitz, R. Benbalagh, R. Delaunay, D. Druart, P. Jonnard, H. Ringuenet, Eur. Phys. J. Appl. Phys. 31, 147 (2005)

23. J.-M. André, P. Jonnard, C. Michaelsen, J. Wiesmann, F. Bridou, M.-F. Ravet, A. Jérôme, F. Delmotte, E.O. Filatova, X-Ray Spectrom. 34, 203 (2005)

24. C. Bonnelle, F. Vergand, P. Jonnard, J.-M. André, P.-F. Staub, A. Avila, P. Chargelègue, M.-F. Fontaine, D. Laporte, P. Paquier, A. Ringuenet, B. Rodriguez, Rev. Sci. Instrum. 65, 3466 (1994)

25. M. Terauchi, M. Koike, K. Fukushima, A. Kimura, J. Electron Microsc. 59, 251 (2010)

26. M. Niibe, T. Tokuhima, N. Takehira, Y. Araki, J. Electron Spectrosc. Relat. Phenom. (2017) (in press), DOI: $10.1016 / \mathrm{j}$.elspec.2017.02.001

27. E. Tegeler, N. Kosuch, G. Wiech, A. Faessler, Phys. Stat. Sol. B 84, 561 (1977) 